\title{
WEIGHTED NORM INEQUALITIES FOR AVERAGING OPERATORS OF MONOTONE FUNCTIONS
}

\author{
C.J. Neugebauer.
}

$\begin{array}{ll}\text { Abstract } & \text { We prove weighted norm inequalities for the averaging operator } A f(x)= \\ & \frac{1}{x} \int_{0}^{x} f \text { of monotone functions. }\end{array}$

\section{Introduction}

This paper is concerned with weighted Hardy type inequalities of the form

$$
\int_{0}^{\infty}\left(\frac{1}{x} \int_{0}^{x} f\right)^{p} w(x) d x \leq c \int_{0}^{\infty} f(x)^{p} v(x) d x .
$$

Muckenhoupt [6] has given necessary and sufficient conditions for $(*)$ to hold for arbitrary $f$.

In their paper [1] Ariño and Muckenhoupt studied the problem when the Hardy-Littlewood maximal operator is bounded on Lorentz spaces and observed that this leads to the study of $(*)$ for non-increasing $f$. There are more weights in this case than for general $f[1]$. They solved the problem for $w=v$ by the condition $B_{p}$, i.e., $w \in B_{p}$ if and only if $\int_{r}^{\infty}\left(\frac{r}{x}\right)^{p} w(x) d x \leq c \int_{0}^{\tau} w(x) d x$, $r>0$. The proof is rather lengthy and first establishes that $B_{p}$ implies $B_{p-\epsilon}$ (Lemma 2.1 of [1]).

The purpose of this paper is

(i) to give a much shorter proof of a somewhat more general version of (*) without $B_{p}$ implies $B_{p-\varepsilon}$,

(ii) to prove then $B_{p}$ implies $B_{p-\epsilon}$ using an iterated version of $(*)$,

(iii) to investigate the reverse inequalities

$$
\int_{0}^{\infty} f(x)^{p} w(x) d x \leq c \int_{0}^{\infty}\left(\frac{1}{x} \int_{0}^{x} f\right)^{p} v(x) d x
$$

(iv) to study the same questions for non-decreasing functions, and finally

(v) to present some properties of $B_{p}$-weights suggested by the analogous properties of $A_{p}$-weights as, e.g. the $A_{1} \cdot A_{1}^{1-p}$ factorization of an $A_{p^{-}}$ weight [3]. 
We point, out that the double weight inequality $(*)$ has been characterized in a recent paper by E. Sawyer [7] for non-increasing functions with the $q$-norm of the averaging operator on the lcft and the $p$-norm on the right. It is also possible to prove some of our rcsults by the methods developed in the paper by D.W. Boyd [2].

Throughout the paper we shall use the following notation. The symbol $f \uparrow$ $(f \downarrow)$ means $f: \mathbb{R}_{+} \rightarrow \mathbb{R}_{+}$non-decreasing (non-increasing). For $f \downarrow$ we define $f^{-1}(t)=\inf \{\tau: f(\tau) \leq t\}$ with an analogous statement for $f \uparrow$. In proving (*) for monotone functions we may restrict ourselves to homeomorphisms since a general monotone function can be approximated by homeomorphisms. For $0<r<\infty$, let $\chi_{r}(x)=\chi_{[0, r]}(x)$ and $\chi^{r}(x)=\chi_{[r, \infty)}(x)$. By a weight $w$ we mean any measurable $w: \mathbb{R}_{+} \rightarrow \mathbb{R}_{+}$.

\section{Non-increasing functions}

For the norm inequalities for the averaging operator $A f(x)=\frac{1}{x} \int_{0}^{x} f$ we need the following lemma.

Lemma 2.1. Let $\varphi \downarrow$ and let $W$ be a weight. Then

$$
\begin{aligned}
& \int_{0}^{\infty} \int_{0}^{\infty} \chi_{\varphi(y)}(x) W(x) d x d y=\int_{0}^{\infty} \varphi^{-1}(x) W(x) d x \\
& \int_{0}^{\infty} \int_{0}^{\infty} \chi^{\varphi(y)}(x)\left(\frac{\varphi(y)}{x}\right){ }^{p} W(x) d x d y \\
& =\int_{0}^{\infty}\left\{\frac{1}{x^{p}} \int_{0}^{x} \varphi^{-1}(u) d\left(u^{p}\right)-\varphi^{-1}(x)\right\} W(x) d x .
\end{aligned}
$$

Proof: (i) We interchange the order of integration and get

$$
\int_{0}^{\infty} \int_{0}^{\varphi^{-1}(x)} W(x) d y d x=\int_{0}^{\infty} \varphi^{-1}(x) W(x) d x .
$$

(ii) The left side is, after interchanging the order of integration,

$$
\int_{0}^{\infty} \int_{\varphi^{-1}(x)}^{\infty} \frac{W(x)}{x^{p}}(\varphi(y))^{p} d y d x
$$

and the inner integral in $y$ is

$$
\begin{aligned}
\int_{\varphi^{-1}(x)}^{\infty}(\varphi(y))^{p} d y & =\int_{0}^{x^{p}} \varphi^{-1}\left(t^{1 / p}\right) d t-x^{p} \varphi^{-1}(x) \\
& =\int_{0}^{x} \varphi^{-1}(u) d\left(u^{p}\right)-x^{p} \varphi^{-1}(x) .
\end{aligned}
$$


This can be seen by comparing areas of the regions under the curve $t=(\varphi(y))^{p}$ or $y=\varphi^{-1}\left(t^{1 / p}\right)$.

Definition. For $\mathrm{I} \leq p<\infty$ and $n$ a positive integer we write $(w, v) \in B(p, n)$ if and only if there is $0<c<\infty$ such that for every choice $0<r_{1}, r_{2}, \cdots, r_{n}<$ $\infty$,

$$
\begin{aligned}
\int_{0}^{\infty}\{ & \left.\prod_{1}^{n}\left(\chi_{r_{j}}(x)+\chi^{r_{j}}(x)\left(\frac{r_{j}}{x}\right)^{p}\right)\right\} w(x) d x \\
& \leq c \int_{0}^{\infty}\left\{\prod_{1}^{n} \chi_{r_{j}}(x)\right\} v(x) d x .
\end{aligned}
$$

Remark. (i) In case $w=v$, we simply write $w \in B(p, n)$.

(ii) If $n=1$, then $(w, v) \in B(p, 1)$ means $\int_{0}^{r} w+\int_{r}^{\infty}\left(\frac{r}{x}\right)^{p} w(x) d x \leq c \int_{0}^{r} v$, $r>0$. Hence, if $v=w$, we get the equivalent condition

$$
\int_{r}^{\infty}\left(\frac{r}{x}\right)^{p} w(x) d x \leq c \int_{0}^{r} w
$$

introduced in $[\mathbf{1}]$ as $B_{p}$.

(iii) The smallest $c$ in the above expressions will be referred to as the $B_{p}(w)$ constant of $w$ or the $B(p, n)$-constant of $(w, v)$.

(iv) If we let $r_{n} \rightarrow \infty$ we see that $B(p, n) \subset B(p, n-1)$.

Theorem 2.2. Let $1 \leq p<\infty$ and let $f_{j} \downarrow, j=1, \cdots, n$. Then

$$
\int_{0}^{\infty}\left\{\prod_{1}^{n}\left(\frac{1}{x} \int_{0}^{x} f_{j}\right)^{p}\right\} w(x) d x \leq c \int_{0}^{\infty}\left\{\prod_{j=1}^{n} f_{j}\left(\frac{1}{x} \int_{0}^{x} f_{j}\right)^{p-1}\right\} v(x) d x
$$

if and only if $(w, v) \in B(p, n)$ with ce equal to the $B(p, n)$-constant of $(w, v)$.

Proof: If $f_{j}=\chi_{r j}, j=1, \cdots, n$, then the norm inequality easily gives $(w, v) \in B(p, n)$. We do the converse for $n=2$; the general case is obtained by repeating the argument.

Let $\varphi_{j} \downarrow, j=1,2$, and let $r_{j}=\varphi_{j}\left(y_{j}\right)$, where $0<y_{1}, y_{2}<\infty$. We next integrate the condition $B(p, n)$ over $\left\{\left(y_{1}, y_{2}\right): y_{1}, y_{2}>0\right\}$ and obtain

$$
\begin{aligned}
L & \equiv \int_{0}^{\infty} \int_{0}^{\infty} \int_{0}^{\infty} \psi_{1}\left(x, y_{1}\right) \psi_{2}\left(x, y_{2}\right) w(x) d x d y_{1} d y_{2} \\
& \leq c \int_{0}^{\infty} \int_{0}^{\infty} \int_{0}^{\infty} \chi_{\varphi_{1}\left(y_{1}\right)}(x) \chi_{\varphi_{2}\left(y_{2}\right)}(x) v(x) d x d y_{1} d y_{2} \equiv R
\end{aligned}
$$


where $\psi_{j}\left(x, y_{j}\right)=\chi_{\varphi_{j}\left(y_{j}\right)}(x)+\chi^{\varphi_{j}\left(y_{j}\right)}(x)\left(\frac{\varphi_{j}\left(y_{j}\right)}{x}\right)^{p}$. By Lermma 2.1,

$$
\begin{aligned}
R & =\int_{0}^{\infty} \int_{0}^{\infty} \varphi_{1}^{-1}(x) \chi_{\varphi_{2}\left(y_{2}\right)}(x) v(x) d x d y_{2} \\
& =\int_{0}^{\infty} \varphi_{1}^{-1}(x) \varphi_{2}^{-1}(x) v(x) d x
\end{aligned}
$$

The inner 2 integrals of $L$ can be written as

$$
\begin{gathered}
\int_{0}^{\infty} \int_{0}^{\varphi_{1}\left(y_{1}\right)} \psi_{2}\left(x, y_{2}\right) w(x) d x d y_{1} \\
+\int_{0}^{\infty} \int_{\varphi_{1}\left(y_{1}\right)}^{\infty} \psi_{2}\left(x, y_{2}\right)\left(\frac{\varphi_{1}\left(y_{1}\right)}{x}\right)^{p} w(x) d x d y_{1}=I_{1}+I_{2} .
\end{gathered}
$$

By (i) of Lemma 2.1 with $W=\psi_{2} w, I_{1}=\int_{0}^{\infty} \varphi_{1}^{-1}(x) \psi_{2}\left(x, y_{2}\right) w(x) d x$. Similarly, by (ii) of Lemma 2.1 ,

$$
I_{2}=\int_{0}^{\infty}\left\{\frac{1}{x^{p}} \int_{0}^{x} \varphi_{1}^{-1}(u) d\left(u^{p}\right)-\varphi_{1}^{-1}(x)\right\} \psi_{2}\left(x, y_{2}\right) w(x) d x
$$

Hence $I_{1}+I_{2}=\int_{0}^{\infty}\left\{\frac{1}{x^{p}} \int_{0}^{x} \varphi_{1}^{-1}(u) d\left(u^{p}\right)\right\} \psi_{2}\left(x, y_{2}\right) u(x) d x$. We integrate this expression in $y_{2}$ and repeat the argument to get

$$
L=\int_{0}^{\infty}\left\{\frac{1}{x^{p}} \int_{0}^{x} \varphi_{1}^{-1}(u) d\left(u^{p}\right)\right\}\left\{\frac{1}{x^{p}} \int_{0}^{x} \varphi_{2}^{-1}(u) d\left(u^{p}\right)\right\} w(x) d x .
$$

We thus obtain

$$
\begin{gathered}
\int_{0}^{\infty}\left\{\frac{1}{x^{p}} \int_{0}^{x} \varphi_{1}^{-1}(u) d\left(u^{p}\right)\right\}\left\{\frac{1}{x^{p}} \int_{0}^{x} \varphi_{2}^{-1}(u) d\left(u^{p}\right)\right\} w(x) d x \\
\leq c \int_{0}^{\infty} \varphi_{1}^{-1}(x) \varphi_{2}^{-1}(x) v(x) d x .
\end{gathered}
$$

We remark here that the constant $c$ is the same as the $c$ in $B(p, 2)$.

We now let $\varphi_{j}^{-1}(u)=f_{j}(u)\left(\frac{1}{u} \int_{0}^{u} f_{j}\right)^{p-1}, j=1,2$, and observe that

$$
\begin{aligned}
\frac{1}{x^{p}} \int_{0}^{x} \varphi_{j}^{-1}(u) d\left(u^{p}\right) & =p \frac{1}{x^{p}} \int_{0}^{x} f_{j}(u)\left(\int_{0}^{u} f_{j}\right)^{p-1} d u \\
= & \frac{1}{x^{p}}\left(\int_{0}^{x} f_{j}\right)^{p} .
\end{aligned}
$$


This completes the proof of Theorem 2.2.

Remark. It may be of interest to point out that there is an easy condition for equality in Theorem 2.2. Let
(i) $\int_{0}^{\infty} A f^{p} w=\int_{0}^{\infty} f A f^{p-1} v$,
(ii) $v(t)=p t^{p-1} \int_{t}^{\infty} \frac{w(x)}{x^{p}} d x$.

If (i) holds for $f \downarrow$, then (ii) follows. Simply let $f=\chi_{t}$ and differentiate the resulting equation $\int_{0}^{t} v=\int_{0}^{t} w+\int_{t}^{\infty}\left(\frac{t}{x}\right)^{p} w(x) d x$. Conversely, if (ii) holds, then (i) is valid for any $f: \mathbb{R}_{+} \rightarrow \mathbb{R}_{+}$. This can be seen by replacing $v$ in (i) by (ii) and then integrating by parts.

We state the special casc $p=1$ of Theorem 2.2 as

Corollary 2.3. If $f_{j} \downarrow, j=1, \cdots, n$, then

$$
\int_{0}^{\infty}\left\{\prod_{1}^{n}\left(\frac{1}{x} \int_{0}^{x} f_{j}\right)\right\} w(x) d x \leq c \int_{0}^{\infty}\left\{\prod_{j=1}^{n} f_{j}(x)\right\} v(x) d x
$$

if and only if $(w, v) \in B(1, n)$.

The case $w=v$ of Theorem 2.2 yields as a special case the AriñoMuckenhoupt weighted norm inequality for non-increasing functions [1].

Corollary 2.4. Let $1 \leq p<\infty$ and $f_{j} \downarrow, j=1, \cdots, n$. Then

$$
\int_{0}^{\infty}\left\{\prod_{j=1}^{n}\left(\frac{1}{x} \int_{0}^{x} f_{j}\right)^{p}\right\} w(x) d x \leq c \int_{0}^{\infty}\left\{\prod_{j=1}^{n} f_{j}(x)^{p}\right\} w(x) d x
$$

if and only if $w \in B(p, n)$.

Proof: The necessity follows from $f_{j}=\chi_{r_{j}}$, and for the sufficiency we apply Theorem 2.2 and use Hölder's inequality to obtain

$$
\begin{gathered}
\int_{0}^{\infty}\left\{\prod_{j=1}^{n} f_{j}\right\} \cdot \prod_{j=1}^{n}\left(\frac{1}{x} \int_{0}^{x} f_{j}\right)^{p-1} w(x) d x \\
\leq\left\{\int_{0}^{\infty}\left\{\prod f_{j}\right\}^{p} w\right\}^{1 / p}\left\{\int_{0}^{\infty}\left\{\prod_{j=1}^{n}\left(\frac{1}{x} \int_{0}^{x} f_{j}\right)^{p}\right\} w\right\}^{1 / p^{\prime}} .
\end{gathered}
$$

Divide by the last factor to obtain the norm inequality.

Remark. (i) For a single weight the conditions $B(p, n)$ and $B_{p}$ are equivalent, i.e., $w \in B(p, n)$ iff $w \in B_{p}$. Since the implication $B(p, n) \subset B_{p}$ was 
already observed in (iv) of the previous remark, we only need to show that $B_{p} \subset B(p, n)$. It is clear that if $u \downarrow$ and $w \in B_{p}$, then $w w \in B_{p}$. Let now $f_{j} \downarrow$, $j=1,2$, and let $w \in B_{p}$. Then $A f_{2}(x)^{p} w(x) \in B_{p}$, and hence

$$
\int_{0}^{\infty} A f_{1}^{p} A f_{2}^{p} w \leq c \int_{0}^{\infty} f_{1}^{p} A f_{2}^{p} w
$$

Since $f_{1}^{p} w \in B_{p}$, we can continue this inequality $\leq c \int_{0}^{\infty} f_{1}^{p} f_{2}^{p} w$, i.e., $w \in$ $B(p, 2)$.

(ii) Results related to the above Corollaries can also be found in [2].

We will now show that an iterated version of Corollary 2.4 provides a short proof of $B_{p}$ implies $B_{p-\epsilon}$, the basic Lemma in [1]. Similar ideas for the HardyLittlewood maximal operator and the " $A_{p}$ implies $A_{p-\epsilon}$ " case can be found in $[4],[5]$.

Theorem 2.5. Let $1 \leq p<\infty$ and let $w \in B(p, 1)$. Then there is $\epsilon>0$ such that $w \in B(p-\epsilon, 1)$.

Proof: Fix $r>0$ and let $f=\chi_{r}$. If $A_{n} f(x)$ is the $n$-times iterated averaging operator, i.e., $A_{0} f(x)=f(x), A_{1} f(x)=\frac{1}{x} \int_{0}^{x} f, \cdots$, then for $n \geq 1$,

$$
A_{n} f(x)=\left\{\begin{array}{l}
1, \quad 0<x \leq r \\
\frac{r}{x} \sum_{j=0}^{n-1} \frac{1}{j !} \log ^{j}\left(\frac{x}{r}\right), \quad x>r .
\end{array}\right.
$$

Since $w \in B(p, 1)$ we have from Corollary 2.4 ,

$$
\begin{aligned}
\int_{0}^{\infty} A_{n} f(x)^{p} w(x) d x & \leq c^{n} \int_{0}^{\infty} f(x)^{p} w(x) d x \\
& =c^{n} \int_{0}^{r} w(x) d x .
\end{aligned}
$$

For $x>r$,

$$
\begin{gathered}
A_{n} f(x)^{p}=\left(\frac{r}{x}\right)^{p}\left(\sum_{j=0}^{n-1} \frac{1}{j !} \log ^{j}\left(\frac{x}{r}\right)\right)^{p} \\
\geq\left(\frac{r}{x}\right)^{p}\left(\sum_{j=0}^{n-1} \frac{1}{j !} \log ^{j}\left(\frac{x}{r}\right)\right) \geq\left(\frac{r}{x}\right)^{p} \frac{1}{(n-1) !} \log ^{n-1}\left(\frac{x}{r}\right),
\end{gathered}
$$

where the next to the last inequality follows since $\sum_{j=0}^{n-1} \geq 1$. We substitute this in our norm inequality and get

$$
\int_{r}^{\infty}\left(\frac{r}{x}\right)^{p} \frac{1}{(n-1) !} \log ^{n-1}\left(\frac{x}{r}\right) w(x) d x \leq c^{n} \int_{0}^{r} w(x) d x .
$$


Let $s>c$. Then

$$
\int_{r}^{\infty}\left(\frac{r}{x}\right)^{p} \sum_{n=1}^{\infty} \frac{1}{(n-1) !}\left(\frac{\log \frac{x}{r}}{s}\right)^{n-1} w(x) d x \leq C \int_{0}^{r} w(x) d x
$$

or

$$
\int_{r}^{\infty}\left(\frac{r}{x}\right)^{p-1 / s} w(x) d x \leq C \int_{0}^{r} w(x) d x
$$

i.e. $w \in B\left(p-\frac{1}{s}, 1\right)$.

\section{The case $n=1$ and reverse inequalities}

We begin by asking for which averaging operator is $(w, v) \in B(p, 1)$ a necessary and sufficient condition for a weighted norm inequality. The case $p=1$ is handled by Corollary 2.3 with $A f(x)=\frac{1}{x} \int_{0}^{x} f$. For $1 \leq p<\infty$ we define

$$
A_{p} f(x)=\left\{\frac{1}{x^{p}} \int_{0}^{x} f(u)^{p} d\left(u^{p}\right)\right\}^{1 / p}
$$

Theorem 3.1. If $f \downarrow$ and $1 \leq p<\infty$, then

$$
\int_{0}^{\infty} A_{p} f(x)^{p} w(x) d x \leq c \int_{0}^{\infty} f(x)^{p} v(x) d x
$$

if and only if $(w, v) \in B(p, 1)$.

Proof: The necessity follows by taking $f=\chi_{r}$.

For the sufficiency simply let $\varphi^{-1}(u)=f(u)^{p}$ in the proof of Theorem 2.2.

We will now characterize the weights $(w, v)$ for which the reverse inequality

$$
\int_{0}^{\infty} f(x)^{p} w(x) d x \leq c \int_{0}^{\infty}\left(\frac{1}{x} \int_{0}^{x} f\right)^{p} v(x) d x
$$

holds for $f \downarrow$.

Theorem 3.2. Let $f \downarrow$ and $1 \leq p<\infty$. Then

$$
\int_{0}^{\infty} f(x)^{p} w(x) d x \leq c \int_{0}^{\infty}\left(\frac{1}{x} \int_{0}^{x} f\right)^{p} v(x) d x
$$


if and only if $\int_{0}^{r} w \leq c\left(\int_{0}^{r} v+\int_{r}^{\infty}\left(\frac{\tau}{x}\right)^{p} v(x) d x\right), r>0$, with the same $c$.

Proof: The necessity follows with $f=\chi_{r}$. For the sufficiency, let $\varphi \downarrow$ and let $r=\varphi(y)$. Then as in the proof of Theorem 2.2,

$$
\int_{0}^{\infty} \int_{0}^{\varphi(y)} w(x) d x d y=\int_{0}^{\infty} \varphi^{-1}(x) w(x) d x
$$

and

$$
\begin{aligned}
& \int_{0}^{\infty} \int_{0}^{\varphi(y)} v(x) d x d y+\int_{0}^{\infty} \int_{\varphi(y)}^{\infty} \frac{w(x)}{x^{p}}(\varphi(y))^{p} d x d y \\
= & \int_{0}^{\infty} \varphi^{-1}(x) v(x) d x+\int_{0}^{\infty} \frac{1}{x^{p}} \int_{0}^{x} \varphi^{-1}(u) d\left(u^{p}\right) v(x) d x \\
- & \int_{0}^{\infty} \varphi^{-1}(x) v(x) d x=\int_{0}^{\infty} \frac{1}{x^{p}} \int_{0}^{x} \varphi^{-1}(u) d\left(u^{p}\right) v(x) d x
\end{aligned}
$$

We let now $\varphi^{-1}(u)=f(u)\left(\frac{1}{u} \int_{0}^{u} f\right)^{p-1}$ and obtain

$$
\int_{0}^{\infty} f(x)\left(\frac{1}{x} \int_{0}^{x} f\right)^{p-1} w(x) d x \leq c \int_{0}^{\infty}\left(\frac{1}{x} \int_{0}^{x} f\right)^{p} v(x) d x
$$

We complete the proof by noting that $\frac{1}{x} \int_{0}^{x} f \geq f(x)$ since $f \downarrow$.

We will now characterize the single weights, i.e., $w=v$, for which the above reverse inequality holds for a given $0<c<1$.

Theorem 3.3. The following statements are equivalent for $f \downarrow, 0<c<1$, $1<p<\infty$, and $w \in L_{\mathrm{loc}}^{1}\left(\mathbb{R}_{+}\right)$.

(1) $\int_{0}^{\infty} f^{p} w \leq c \int_{0}^{\infty} A f^{p} w$

(2) $B_{p^{\prime}}\left(w\left(y^{1-p^{\prime}}\right)\right) \leq \frac{c}{1-c}$.

Proof: (1) $\rightarrow$ (2). If $f=\chi_{r}$ we get

$$
\int_{0}^{r} w \leq c\left(\int_{0}^{r} w+\int_{r}^{\infty}\left(\frac{r}{x}\right)^{p} w(x) d x\right) .
$$

We let $x=y^{1-p^{\prime}}$ and get

$$
\begin{gathered}
\int_{0}^{r} w(x) d x=\left(p^{\prime}-1\right) \int_{r^{1-p}}^{\infty} w\left(y^{1-p^{\prime}}\right) \frac{d y}{y^{p^{\prime}}} \\
r^{p} \int_{r}^{\infty} \frac{w(x)}{x^{p}} d x=\left(p^{\prime}-1\right) r^{p} \int_{0}^{r^{1-p}} w\left(y^{1-p^{\prime}}\right) d y .
\end{gathered}
$$


Hence

$$
(1-c)\left(p^{\prime}-1\right) \int_{r^{1-p}}^{\infty} w\left(y^{1-p^{\prime}}\right) \frac{d y}{y^{p^{\prime}}} \leq c\left(p^{\prime}-1\right) r^{p} \int_{0}^{r^{1-p}} w\left(y^{1-p^{\prime}}\right) d y .
$$

If we set $\rho=r^{1-p}$, then $r^{p}=\frac{1}{\rho^{p^{\prime}}}$ and (2) follows.

(2) $\rightarrow(1)$. We have

$$
\int_{r}^{\infty}\left(\frac{r}{y}\right)^{p^{\prime}} w\left(y^{1-p^{\prime}}\right) d y \leq \frac{c}{1-c} \int_{0}^{r} w\left(y^{1-p^{\prime}}\right) d y .
$$

Let $y=x^{1-p}$. Then, again

$$
\begin{gathered}
\int_{r}^{\infty}\left(\frac{r}{y}\right)^{p^{\prime}} w\left(y^{1-p^{\prime}}\right) d y=r^{p^{\prime}}(p-1) \int_{0}^{r^{1-p^{\prime}}} w(x) d x \\
\int_{0}^{r} w\left(y^{1-p^{\prime}}\right) d y=(p-1) \int_{r^{1-p^{\prime}}}^{\infty} \frac{w(x)}{x^{p}} d x .
\end{gathered}
$$

Thus, with $\rho=r^{1-p^{\prime}}$ we get

$$
\int_{0}^{\rho} w(x) d x \leq \frac{c}{1-c} \int_{\rho}^{\infty}\left(\frac{\rho}{x}\right)^{p} w(x) d x .
$$

We add $\frac{c}{1-c} \int_{0}^{\rho} w$ to both sides and get

$$
\int_{0}^{\rho} w \leq c\left(\int_{0}^{\rho} w+\int_{\rho}^{\infty}\left(\frac{\rho}{x}\right)^{p} w(x) d x\right) .
$$

Apply now Theorem 3.2 .

Remark. (2) of Theorem 3.3 reminds one of the duality $w \in A_{p}$ iff $w^{1-p^{\prime}} \in$ $A_{p^{\prime}}$.

\section{Non-decreasing functions}

We will not dwell on the straightforward results of $f \uparrow$ that one gets from our previous results via the change of variables $x \rightarrow \frac{1}{x}$. In particular we have

Theorem 4.1. If $f \uparrow$ and $1 \leq p<\infty$, then

$$
\int_{0}^{\infty}\left(x \int_{x}^{\infty} f(u) \frac{d u}{u^{2}}\right)^{p} w(x) d x \leq c \int_{0}^{\infty} f(x)^{p} w(x) d x
$$

if and only if $\int_{0}^{r}\left(\frac{x}{r}\right)^{p} w(x) d x \leq c \int_{r}^{\infty} w(x) d x, r>0$.

In order to see what type of results one has for the averaging operator $\frac{1}{x} \int_{0}^{x} f$ for $f \uparrow$ we need a lemma similar to Lemma 2.1 . 
Lemma 4.2. Let $\varphi \nmid$ with $\varphi(0)=0$, and let $W$ be a weight. Then

$$
\begin{gathered}
\int_{0}^{\infty} \int_{0}^{\infty} \chi^{\varphi(y)}(x) W(x) d x d y=\int_{0}^{\infty} \varphi^{-1}(x) W(x) d x \\
\int_{0}^{\infty} \int_{0}^{\infty} \chi^{\varphi(y)}(x)\left(\frac{x-\varphi(y)}{x}\right)^{p} W(x) d x d y \\
=\int_{0}^{\infty}\left\{\frac{1}{x^{p}} \int_{0}^{x} \varphi^{-1}(x-u) d\left(u^{p}\right)\right\} W(x) d x
\end{gathered}
$$

Proof: For (i) we simply interchange the order of integration. The left side of (ii) is $\int_{0}^{\infty} \int_{0}^{\varphi^{-1}(x)} \frac{W(x)}{x^{p}}(x-\varphi(y))^{p} d y d x$ and the inner integral is the same as

$$
\int_{0}^{x^{p}} \varphi^{-1}\left(x-t^{1 / p}\right) d t=\int_{0}^{x} \varphi^{-1}(x-u) d\left(u^{p}\right)
$$

as can be seen by interpreting the integral as area under $t=(x-\varphi(y))^{p}$.

Definition. Let $n$ be a positive integer and $1 \leq p<\infty$. We say that $(w, v) \in C(p, n)$ if and only if there is $0<c<\infty$ such that for every choice $0<r_{1}, r_{2}, \cdots, r_{n}<\infty$,

$$
\int_{0}^{\infty}\left\{\prod_{j=1}^{n} \chi^{r_{j}}(x)\right\} w(x) d x \leq c \int_{0}^{\infty}\left\{\prod_{j=1}^{n} \chi^{r_{j}}(x)\left(\frac{x-r_{j}}{x}\right)^{p} v(x) d x .\right.
$$

Theorem 4.3. Let $f_{j} \uparrow, j=1, \cdots, n$. Then

$$
\int_{0}^{\infty}\left\{\prod_{1}^{n} f_{j}(x)\right\} w(x) d x \leq c \int_{0}^{\infty}\left\{\prod_{1}^{n}\left(\frac{1}{x} \int_{0}^{x} f_{j}\right)\right\} v(x) d x
$$

if and only if $(w, v) \in C(1, n)$.

Proof: The necessity follows by taking $f_{j}=\chi^{r_{j}}$. As in Theorem 2.2 we prove the converse for $n=2$; the general case is obtained by repeating the argument. We let $\varphi_{j} \uparrow, \varphi_{j}(0)=0$, and $r_{j}=\varphi_{j}\left(y_{j}\right), j=1,2$, where $0<y_{1}, y_{2}<\infty$. We next integrate the $C(1, n)$ condition over all such $\left(y_{1}, y_{2}\right)$ and obtain

$$
\begin{aligned}
& L \equiv \int_{0}^{\infty} \int_{0}^{\infty} \int_{0}^{\infty} \chi^{\varphi_{1}\left(y_{1}\right)}(x) \chi^{\varphi_{2}\left(y_{2}\right)}(x) w(x) d x d y_{1} d y_{2} \\
& \leq c \int_{0}^{\infty} \int_{0}^{\infty} \int_{0}^{\infty} \psi_{1}\left(x, y_{1}\right) \psi_{2}\left(x, y_{2}\right) v(x) d x d y_{1} d y_{2} \equiv R
\end{aligned}
$$


where $\psi_{j}\left(x, y_{j}\right)=\chi^{\varphi_{j}\left(y_{j}\right)}(x)\left(\frac{x-\varphi_{j}\left(y_{j}\right)}{x}\right)$. By (i) of Lemma 4.2,

$$
L=\int_{0}^{\infty} \varphi_{2}^{-1}(x) \varphi_{1}^{-1}(x) w(x) d x
$$

and by (ii) with $p=1$,

$$
R=\int_{0}^{\infty}\left(\frac{1}{x} \int_{0}^{x} \varphi_{1}^{-1}\right)\left(\frac{1}{x} \int_{0}^{x} \varphi_{2}^{-1}\right) v(x) d x
$$

From this we get the theorem by letting $\varphi_{j}^{-1}(t)=f_{j}(t)$ if $f_{j}(0)=0$. Otherwise, let $\epsilon_{n}(x)=n x$, if $0 \leq x \leq \frac{1}{n}$, and $\epsilon_{n}(x)=1, x>\frac{1}{n}$. If $\varphi_{j, n}^{-1}(t)=$ $\epsilon_{n}(t) f_{j}(t)$, then we get the weighted norm inequality for $\epsilon_{n} f_{j}$, and the final result by letting $n \rightarrow \infty$.

Corollary 4.4. Let $f \uparrow$ and $n$ a positive integer. Then

$$
\int_{0}^{\infty} f(x)^{n} w(x) d x \leq c \int_{0}^{\infty}\left(\frac{1}{x} \int_{0}^{x} f\right)^{n} v(x) d x
$$

if and only if $(w, v) \in C(1, n)$.

Proof: If $(w, v) \in C(1, n)$, then the inequality follows from Theorem 4.3 by letting $f_{1}=f_{2}=\cdots=f_{n_{2}}$. Conversely, let $f=\prod_{1}^{n} \chi^{r_{j}}$. Then $f=f^{n}$ and by Hölder's inequality

$$
\left(\frac{1}{x} \int_{0}^{x} f\right)^{n} \leq \prod_{1}^{n}\left(\frac{1}{x} \int_{0}^{x} \chi^{r_{j}}\right)=\prod_{1}^{n} \chi^{r_{j}}(x)\left(\frac{x-r_{j}}{x}\right) .
$$

Remark. We were unable to find a characterization of

$$
\int_{0}^{\infty} f(x)^{p} w(x) d x \leq c \int_{0}^{\infty}\left(\frac{1}{x} \int_{0}^{x} f\right)^{p} v(x) d x
$$

for $f \uparrow$ and $p$ not a positive integer. However, as we shall see, $(w, v) \in C(p, 1)$ controls the averaging operator

$$
A_{p} f(x)=\frac{1}{x^{p}} \int_{0}^{x} f(x-u) d\left(u^{p}\right) .
$$

We observe that, when $p$ is a positive integer, then $\int_{0}^{x} f(x-u) d\left(u^{p}\right)$ is, apart from a multiplicative constant, the p-times iterated integral of $f$. 
Theorem 4.5. Let $f \uparrow$ and $1 \leq p<\infty$. Then

(i) $\int_{0}^{\infty} A_{p} f(x) w(x) d x \leq c \int_{0}^{\infty} f(x) v(x) d x$ if and only if $\int_{r}^{\infty}\left(\frac{x-r}{x}\right)^{p} w(x)$ $d x \leq c \int_{r}^{\infty} v(x) d x, r>0$.

(ii) $\int_{0}^{\infty} f(x) w(x) d x \leq c \int_{0}^{\infty} A_{p} f(x) v(x) d x$ if and only if $\int_{r}^{\infty} w(x) d x \leq$ $c \int_{r}^{\infty}\left(\frac{x-r}{x}\right)^{p} v(x) d x, r>0$, i.e., $(w, v) \in C(p, 1)$.

Proof: (i) For the necessity let $f=\chi^{\tau}$. To prove the sufficiency, let $\varphi \uparrow$, $\varphi(0)=0$, and $r=\varphi(y), 0<y<\infty$. Then

$$
L \equiv \int_{0}^{\infty} \int_{\varphi(y)}^{\infty} \frac{w(x)}{x^{p}}(x-\varphi(y))^{p} d x d y \leq c \int_{0}^{\infty} \int_{\varphi(y)}^{\infty} v(x) d x d y \equiv R .
$$

By Lemma $4.2, R=\int_{0}^{\infty} \varphi^{-1}(x) v(x) d x$ and

$$
L=\int_{0}^{\infty}\left\{\frac{1}{x^{p}} \int_{0}^{x} \varphi^{-1}(x-u) d\left(u^{p}\right)\right\} w(x) d x .
$$

The proof can be completed by letting $\varphi^{-1}(x)=f(x)$ if $f(0)=0$; otherwise let $\varphi^{-1}(x)=\epsilon_{n}(x) f(x)$ as in the proof of Theorem 4.3 .

The proof of (ii) is the same as the one for (i)

\section{More properties of weights}

We begin with a "change of variables" result for $B_{p}$-weights.

Theorem 5.1. . If $1<q<p<\infty$ and $w \in B_{q}$, then $w\left(x^{\frac{p-1}{q-1}}\right) \in B_{p}$.

Proof: We set $I_{r}=\int_{r}^{\infty}\left(\frac{r}{x}\right)^{p} w\left(x^{\frac{p-1}{q-1}}\right) d x$ and let $u=x^{\alpha}, \alpha=\frac{p-1}{q-1}$. Then

$$
\begin{aligned}
I_{r} & =c \int_{r^{\alpha}}^{\infty}\left(\frac{r}{u^{1 / \alpha}}\right)^{p} w(u) u^{\frac{1-\alpha}{\alpha}} d u \\
& =c \int_{r^{\alpha}}^{\infty} \frac{r^{p}}{u^{(p+\alpha-1) / \alpha}} w(u) d u .
\end{aligned}
$$

We observe that $(p+\alpha-1) / \alpha=q$ and so

$$
I_{r}=\int_{r^{\alpha}}^{\infty}\left(\frac{r^{\alpha}}{u}\right)^{q} w(u) d u \cdot r^{p-\alpha q} .
$$


Since $w \in B_{q}$ and $p-\alpha q=\frac{q-p}{q-1}<0$, we see that

$$
\begin{gathered}
I_{r} \leq c r^{\frac{q-p}{\alpha-1}} \int_{0}^{r^{\alpha}} w(u) d u=c r^{1-\alpha} \int_{0}^{r} w\left(x^{\alpha}\right) x^{\alpha-1} d x \\
\leq c \int_{0}^{r} w\left(x^{\alpha}\right) d x .
\end{gathered}
$$

The case $q=1$ yields a slightly stronger result, which we state as

Theorem 5.2. If $w \in B_{1}$ and $\alpha \geq 1$, then $w\left(x^{\alpha}\right) \in B_{1}$ with $B_{1}(w)=$ $B_{1}\left(w\left(x^{\alpha}\right)\right)$

Proof: If $I_{r}=\int_{r}^{\infty}\left(\frac{r}{x}\right) w\left(x^{\alpha}\right) d x$ and $u=x^{\alpha}$, then

$$
\begin{gathered}
I_{r}=\frac{I}{\alpha} \int_{r^{\alpha}}^{\infty}\left(\frac{r}{u^{1 / \alpha}}\right) w(u) u^{1 / \alpha^{-1}} d u=\frac{r^{1-\alpha}}{\alpha} \int_{r^{\alpha}}^{\infty}\left(\frac{r^{\alpha}}{u}\right) w(u) d u \\
\leq c r^{1-\alpha} \int_{0}^{r} w\left(x^{\alpha}\right) x^{\alpha-1} d x \leq c \int_{0}^{r} w\left(x^{\alpha}\right) d x
\end{gathered}
$$

since $\alpha \geq 1$.

The next result reminds one of the important $A_{p}$-property, i.e., $w \in A_{p} \rightarrow$ $w^{\tau} \in A_{p}$ for some $\tau>1$.

Theorem 5:3. If $w \in B_{p}$, then there is $\epsilon>0$ such that $x^{\epsilon} w\left(x^{1+c}\right) \in B_{p}$.

Proof: Choose $\epsilon>0$ so that $w \in B_{p / 1+\epsilon}$ (Theorem 2.5), and note that

$$
\begin{gathered}
\int_{r}^{\infty}\left(\frac{r}{x}\right)^{p} x^{\epsilon} w\left(x^{1+\epsilon}\right) d x=\frac{1}{1+\epsilon} \int_{r^{1+\varepsilon}}^{\infty} \frac{r^{p}}{u^{p / 1+\epsilon}} w(u) d u \\
=\frac{1}{1+\epsilon} \int_{r^{1+\epsilon}}^{\infty}\left(\frac{r^{1+\epsilon}}{u}\right)^{p / 1+\epsilon} w(u) d u \leq \frac{c}{1+\epsilon} \int_{0}^{r^{1+\epsilon}} w(u) d u \\
=c \int_{0}^{r} x^{\epsilon} w\left(x^{1+\epsilon}\right) d x .
\end{gathered}
$$

Corollary 5.4. If $w \in B_{p}$, then there is $\epsilon>0$ such that $w\left(x^{1+\epsilon}\right) \in B_{p}$.

We are now ready to present a factorization theorem for $B_{p}$-weights similar to the factorization of $w \in A_{p}$ as $w=u v^{1-p}, u, v \in A_{1}$. 
Theorem 5.5. The following statements are equivalent for $1<p<\infty$.

(1) $w \in B_{p}$

(2) $w(x)=u(x) \cdot x^{p-1}$ with $u\left(x^{1 / p}\right) \in B_{1}$.

Proof: $(1) \rightarrow(2)$. All we need to show is that $\frac{w\left(x^{1 / p}\right)}{x^{1 / p^{\prime}}} \equiv u\left(x^{1 / p}\right)$ is in $B_{1}$, and this follows from

$$
\begin{gathered}
\int_{r}^{\infty}\left(\frac{r}{x}\right) \frac{w\left(x^{1 / p}\right)}{x^{1 / p^{\prime}}}=c \int_{r^{1 / p}}^{\infty}\left(\frac{r}{t^{p}}\right) \frac{w(t)}{t^{p / p^{p}}} t^{p-1} d t \\
=c \int_{r^{1 / p}}^{\infty}\left(\frac{r^{1 / p}}{t}\right)^{p} w(t) d t \leq c \int_{0}^{r^{1 / p}} w(t) d t=c \int_{0}^{r} w\left(t^{1 / p}\right) / t^{1 / p^{\prime}} d t .
\end{gathered}
$$

$(2) \rightarrow(1)$. This is simply

$$
\begin{gathered}
\int_{r}^{\infty}\left(\frac{r}{x}\right)^{p} u(x) x^{p-1} d x=\frac{1}{p} \int_{r^{p}}^{\infty}\left(\frac{r}{t^{1 / p}}\right)^{p} u\left(t^{1 / p}\right) d t \\
=\frac{1}{p} \int_{r p}^{\infty}\left(\frac{r^{p}}{t}\right) u\left(t^{1 / p}\right) d t \leq \frac{c}{p} \int_{0}^{r^{p}} u\left(t^{1 / p}\right) d t= \\
c \int_{0}^{r} u(x) x^{p-1} d x .
\end{gathered}
$$

Remark. By Theorem 5.2, if $u\left(x^{1 / p}\right) \in B_{1}$, then $u(x) \in B_{1}$. Thus (2) can be written as $w=u \cdot\left(\frac{1}{x}\right)^{1-p}$, with $u \in B_{1}$. It is also clear that $\frac{1}{x} \in B_{1}$.

\section{Weak type weights}

We say that $w \in R_{p}$ iff $w\left\{A \chi_{r}>y\right\} \leq \frac{c}{y^{p}} \int_{0}^{r} w, r>0$, and we say that

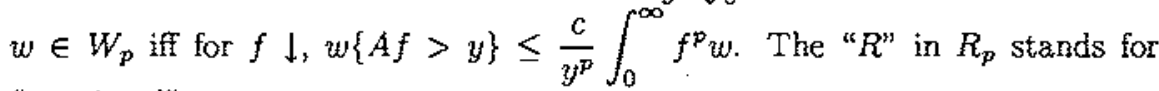
"restricted".

We will study relationships among $R_{p}, W_{p}$, and $B_{p}$, and give a characterization of $B_{p}$.

Theorem 6.1. $w \in R_{p}$ iff there is $0<c<\infty$ so that for $0<r<s<\infty$,

$$
\frac{1}{s^{p}} \int_{0}^{s} w \leq c \frac{1}{r^{p}} \int_{0}^{r} w
$$


Proof: First assume that $w \in R_{p}$. The set $\left\{A \chi_{r}>y\right\}=\left(0, x_{0}\right)$, where $\frac{r}{x_{0}}=y, 0<y<1$. Hence $\int_{0}^{r / y} w \leq \frac{c}{y^{p}} \int_{0}^{r} w$ from which

$$
\frac{1}{s^{p}} \int_{0}^{s} w \leq \frac{c}{r^{p}} \int_{0}^{r} w, \quad s=\frac{r}{y}>r
$$

Conversely, for $0<y<1$, with the same notation as above,

$$
\begin{aligned}
w\left\{A \chi_{r}>y\right\} & =\int_{0}^{x_{0}} w=\frac{1}{y^{p}}\left(\frac{r}{x_{0}}\right)^{p} \int_{0}^{x_{0}} w \\
& \leq \frac{c}{y^{p}} \int_{0}^{\tau} w .
\end{aligned}
$$

The next result shows how $R_{p}$ and $B_{q}$ are related.

Theorem 6.2. If $w \in R_{p}$, then $w \in B_{q}$ for $q>p$.

Proof: From Theorem 6.1, for $s>r$,

$$
\left(\frac{r}{s}\right)^{p} \int_{0}^{s} w \leq c \int_{0}^{r} w
$$

Let $t=\frac{r}{s}$. Then $t^{p} \int_{0}^{r / \ell} w \leq c \int_{0}^{r} w$, or, if $0<\epsilon<1$,

$$
t^{p-\epsilon} \int_{0}^{r / t} w \leq c t^{-\epsilon} \int_{0}^{r} w, \quad 0<t \leq 1
$$

Hence

$$
L \equiv \int_{0}^{1} t^{p-\epsilon} \int_{0}^{r / t} w(x) d x d t \leq c_{\epsilon} \int_{0}^{r} w .
$$

We interchange the order of integration and see that

$$
L \geq \int_{r}^{\infty} \int_{0}^{r / x} w(x) t^{p-\epsilon} d t d x=c \int_{r}^{\infty} w(x)\left(\frac{T}{x}\right)^{p+1-\epsilon} d x .
$$

Thus $w \in B_{q}, q=p+1-\epsilon$.

Example. Let $w(x)=x$. Then $w \in R_{2}$ but not in $W_{2}$ and thus not in $B_{2}$. For let $f(x)=\frac{1}{x \log \frac{1}{x}} \cdot \chi_{\frac{1}{e}}(x)$. Then $w\{A f>y\}=\infty$, but $\int f^{2} w=$ $\int_{0}^{1 / e} \frac{d x}{x \log ^{2} \frac{1}{x}}<\infty$

We will now show that the condition of Theorem 6.1 which characterizes $R_{p}$ will, if slightly modified, characterize $B_{p}$. We begin with 
Lemma 6.3. Assume there exists $1<a<\infty$ and $0<c=c_{a}<1$ such that $\frac{1}{(a x)^{p}} \int_{0}^{a x} w \leq c \frac{1}{x^{p}} \int_{0}^{x} w, x>0$. Then $w \in B_{p}$.

Proof: For $0<N<\infty$, let $w_{N}=w \chi_{N}$. Then $w_{N}$ satisfies the same hypothesis as $w$ with a constant $c=\max \left(c_{a}, 1 / a^{p}\right)<1$.

We have then $\frac{1}{a^{p} x^{p+1}} \int_{0}^{a x} w_{N} \leq \frac{c}{x^{p+1}} \int_{0}^{x} w_{N}$. Hence for $0<r<\infty$ fixed,

$$
L \equiv \frac{1}{a^{p}} \int_{r}^{\infty} \frac{1}{x^{p+1}} \int_{0}^{a x} w_{N}(t) d t d x \leq c \int_{r}^{\infty} \frac{1}{x^{p+1}} \int_{0}^{x} w_{N}(t) d t d x \equiv R .
$$

We interchange the order of integration and see that

$$
\begin{gathered}
L \geq \frac{1}{a^{p}} \int_{a \tau}^{\infty} \int_{t / a}^{\infty} w_{N}(t) \frac{d x}{x^{p+1}} d t=\frac{1}{p} \int_{a r}^{\infty} \frac{w_{N}(t)}{t^{p}} d t, \\
R=c\left\{\int_{0}^{r} \int_{r}^{\infty} w_{N}(t) \frac{d x}{x^{p+1}} d t+\int_{r}^{\infty} \int_{t}^{\infty} w_{N}(t) \frac{d x}{x^{p+1}} d t\right\} \\
=c\left\{\frac{1}{p} \int_{0}^{r} \frac{w_{N}(t)}{r^{p}}+\frac{1}{p} \int_{r}^{\infty} \frac{w_{N}(t)}{t^{p}} d t\right\} .
\end{gathered}
$$

The last integral $\int_{r}^{\infty} \frac{w_{N}(t)}{t^{p}} d t=\left(\int_{r}^{a r}+\int_{a r}^{\infty}\right) \frac{w_{N}(t)}{t^{p}} d t \leq \frac{1}{r^{p}} \int_{r}^{a r} w_{N}(t) d t+$ $\int_{a r}^{\infty} \frac{w_{N}(t)}{t^{p}} d t$. Hence $R \leq c\left\{\frac{1}{p} \int_{0}^{a r} \frac{w_{N}(t)}{r^{p}} d t+\frac{1}{p} \int_{a r}^{\infty} \frac{w_{N}(t)}{t^{p}} d t\right\}$.

From this we obtain,

or

$$
\frac{I}{p}(1-c) \int_{a r}^{\infty} \frac{w_{N}(t)}{t^{p}} d t \leq \frac{c}{p r^{p}} \int_{0}^{a r} w_{N}(t) d t
$$

$$
\int_{a r}^{\infty}\left(\frac{a r}{t}\right)^{p} w_{N}(t) d t \leq \frac{c a^{p} \cdot p}{1-c} \int_{0}^{a r} w_{N}(t) d t .
$$

We complete the proof by letting $N \rightarrow \infty$.

Theorem 6.4. Assume that $w \in L_{\mathrm{loc}}^{1}\left(\mathbb{B}_{+}\right)$. Then $w \in B_{p}$ iff $0<\epsilon<1$ implies the existence of $a_{\epsilon}>1$ such that for $x>0$,

$$
\frac{1}{a^{p} x^{p}} \int_{0}^{a x} w \leq \epsilon \frac{1}{x^{p}} \int_{0}^{x} w, \quad a \geq a_{\epsilon} .
$$

Proof: By Lemma 6.3 we only need to prove the necessity. By Theorem 2.5, there is $\eta>0$ such that $w \in B_{p-\eta}$. Thus for $a>1$,

$$
\frac{\frac{1}{a^{p} x^{p}} \int_{0}^{a x} w}{\frac{1}{x^{p}} \int_{0}^{x} w}=\frac{\frac{1}{(a x)^{p-\eta}} \int_{0}^{a x} w}{\frac{1}{x^{p-\eta}} \int_{0}^{x} w} \cdot\left(\frac{I}{a}\right)^{\eta} .
$$

Since $w \in B_{p-\eta} \subset R_{p-\eta}$, by Theorem 6.1 the first factor $\leq c$ and the proof is complete.

As an application of Theorem 6.4 we will prove 
Theorem 6.5. Let $w \in B_{p}$ and $W(x)=\int_{0}^{x} w$. Then for $0<\alpha<\infty$, $W^{\alpha} \in B_{\alpha p+1}$.

Proof: We do $\alpha=1$ first. Let $0<\epsilon<\frac{1}{p+1}$. Then for $a \geq a_{c}>1$ we have $\frac{x^{p}}{(a r)^{p}} \int_{0}^{a r} w \leq \epsilon \int_{0}^{x} w=\epsilon W(x), 0<x \leq r$. Thus

$$
L \equiv \int_{0}^{r} \frac{x^{p}}{(a r)^{p}} \int_{0}^{a r} w \leq \epsilon \int_{0}^{r} W(x) d x
$$

and

$$
\begin{aligned}
L & =\frac{1}{(p+1)} \frac{r^{p+1}}{(a r)^{p}} W(a r)=\frac{1}{(p+1)} \frac{1}{a^{p+1}}(a r) W(a r) \\
& \geq \frac{1}{p+1} \frac{1}{a^{p+1}} \int_{0}^{a r} W
\end{aligned}
$$

and so $W \in B_{p+1}$.

For the general case, since

$$
W^{\alpha}(x)=\alpha \int_{0}^{x} W^{\alpha-1} w
$$

we only need to verify that $W^{\alpha-1} w \in B_{\alpha p}$. For some $0<c<1$ and $a>1$ we have

$$
\begin{aligned}
\frac{1}{a^{p \alpha}} \int_{0}^{a x} W^{\alpha-1} w & =\frac{1}{\alpha a^{p \alpha}} W^{\alpha}(a x) \leq \frac{1}{\alpha} c W^{\alpha}(x) \\
& =c \int_{0}^{x} W^{\alpha-1} w .
\end{aligned}
$$

\section{The equality $w_{p}=B_{p}$}

In this final section we will prove that $W_{p}=B_{p}$ for $1<p<\infty$, a situation quite analogous to the $A_{p}$-case. I am indebted to Richard Bagby for the original proof of this property. We will present a somewhat simplified version based on some of our previous results. For the definitions of $R_{p}, W_{p}$ see the beginning of section 6 .

Lemma 7.1. Let $w \in R_{p}, 0<a<\infty$, and $1<s<\infty$. Then

$$
\int_{a}^{a s}\left(\frac{a}{u}\right)^{p} w(u) d u \leq c(1+\log s) \int_{0}^{a} w
$$


Proof: We know that by Theorem 6.1,

$$
\frac{1}{t^{p}} \int_{0}^{t a} w \leq c \int_{0}^{a} w, \quad t \geq 1
$$

Hence $L \equiv \int_{1}^{s} \frac{1}{t^{p+1}} \int_{0}^{t a} w \leq c \log s \int_{0}^{a} w$. We interchange the order of integration and get

$$
L \geq \int_{a}^{a s} \int_{u / a}^{s} w(u) \frac{d t}{t^{p+1}} d u=\frac{1}{p} \int_{a}^{a s} w(u)\left[\left(\frac{a}{u}\right)^{p}-\frac{1}{s^{p}}\right] d u .
$$

Hence

$$
\begin{aligned}
\frac{1}{p} \int_{a}^{a s} w(u) & \left(\frac{a}{u}\right)^{p} d u \leq c \log s \int_{0}^{a} w+\frac{1}{p} \frac{1}{s^{p}} \int_{a}^{a s} w \\
& \leq c \log s \int_{0}^{a} w+c \int_{0}^{a} w,
\end{aligned}
$$

since $w \in R_{p}$

Theorem 7.2. $W_{p}=B_{p}$ for $1<p<\infty$.

Proof: The inclusion $B_{p} \subset W_{p}$ is obvious, and for the reverse inclusion we consider for $s>1$ the function $f(x)=1,0 \leq x \leq a ;=a / x, a \leq x \leq s a$; and $=0, x>s a$. Then $A f(a s)=\frac{1+\log s}{s}$. Since $w \in W_{p}$ we have that

$$
w\{A f(x)>y\} \leq \frac{c}{y^{p}} \int_{0}^{\infty} f^{p} w
$$

If $y=\frac{1+\log s}{s}$, we get

$$
\left(\frac{1+\log s}{s}\right)^{p} \int_{0}^{a s} w \leq c\left(\int_{0}^{a} w+\int_{a}^{a s}\left(\frac{a}{u}\right)^{p} w(u) d u\right) \leq c(1+\log s) \int_{0}^{a} w
$$

by Lemma 7.1. Thus

$$
\frac{1}{s^{p}} \int_{0}^{s a} w \leq c(1+\log s)^{1-p} \int_{0}^{a} w
$$

We choose $s$ so large that $c(1+\log s)^{1-p}<1$ and apply Theorem 6.4. 


\title{
References
}

1. M. ARIÑo AND B. MUCKEnhoUPt, Maximal functions on classical Lorentz spaces and Hardy's inequality with weights for non-increasing functions, Trans. Amer. Math. Soc. 320 (1990), 727-735.

2. D.W. BOYD, The Hilbert transform on rearrangement-invariant spaces, Can. Journal Math. 19 (1967), 599-616.

3. J. Garcia-Cuerva and J.L. Rubio de Francia, "Weighted norm inequalities and related topics," North Holland Math Studies 116, North Holland.

4. M.A. Leckband and C.J. Neugebauer, A general maximal operator and the $A_{p}$-condition, Trons. Amer. Math. Soc. 275 (1982), 821-831.

5. M.A. LECKLAND AND C.J. NeugEBAUER, Weighted iterates and variants of the Hardy-Littlewood maximal operator, Trans. Amer. Math. Soc. 279 (1982), 51-61.

6. B. Muckenhoupt, Hardy's inequality with weights, Studia Math $\mathbf{4 4}$ (1972), 31-38.

7. E. SAWYER, Boundedness of Classical Operators on Classical Lorentz spaces, preprint.

\author{
Department of Mathematics \\ Purdue University \\ West Lafayette IN 47907 \\ U.S.A.
}

Primera versió rebuda el 9 d'Octubre de 1989 , darrera versió rebuda el 21 de Maig de 1990 